\title{
Comment on "Strontium clusters: Many-body potential, energetics, and structural transitions" [J. Chem. Phys. 115, 3640 (2001)]
}

\author{
Jonathan P. K. Doye \\ University Chemical Laboratory, Lensfield Road, Cambridge CB2 1EW, United Kingdom \\ Florent Calvo \\ Laboratoire de Physique Quantique, IRSAMC, Université Paul Sabatier, \\ 118 Route de Narbonne, F31062 Toulouse Cedex, France
}

In an interesting recent combined experimental and theoretical paper by Wang et al. on strontium clusters, the experimental magic numbers at $N=34$ and 61 were assigned to the two structures shown in Fig. 11 $\frac{1}{1}$ An additional strong magic number at $N=82$ remained unassigned. As both the assigned $\mathrm{Sr}_{34}$ and $\mathrm{Sr}_{61}$ structures are polytetrahedral (that is the whole space occupied by the cluster can naturally be divided into tetrahedra with atoms at the vertices) it seemed to us that a natural interpretation of the magic number at $N=82$ might be in terms of the third structure in Fig. 10 which was recently suggested as a particularly stable polytetrahedral cluster 2

The assignment of the $\mathrm{Sr}_{34}$ and $\mathrm{Sr}_{61}$ structures was based on calculations using a model potential that Wang et al. parameterized. $\stackrel{\underline{1}}{ }$ However, the assigned structures were not lowest in energy, but calculations of the free energies of these structures (within the harmonic approximation) indicated that they became most stable at $T=129$ and $220 \mathrm{~K}$, respectively.

To test our hypothesis for $\mathrm{Sr}_{82}$ we decided to look at the structure and thermodynamics of this cluster using the same potential as in Ref. 1. As for $\mathrm{Sr}_{34}$ and $\mathrm{Sr}_{61}$ the global minimum of this cluster is not polytetrahedral, but based on a Mackay icosahedron. However, using parallel tempering, a method which has proved particularly useful for probing low-temperature solid-solid transitions in clusters where achieving ergodicity can be particularly challenging, we found no evidence that the 82 -atom polytetrahedral structure depicted in Fig. [1 est in free energy.

This failure led us to check the thermodynamics of $\mathrm{Sr}_{34}$ and $\mathrm{Sr}_{61}$ presented in Ref. 1 using parallel tempering. The resulting heat capacities (Fig. 21) are very different from Ref. 1, showing no evidence of large peaks in the heat capacity at the expected transition temperatures.

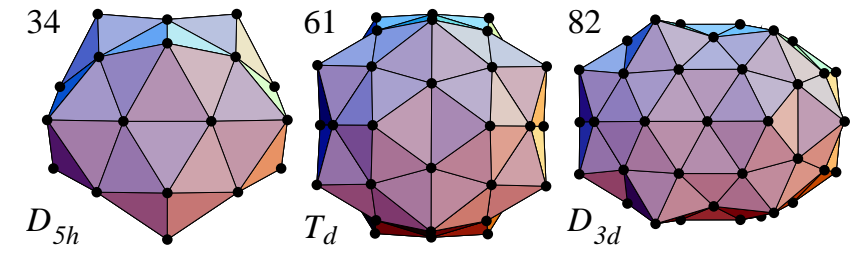

FIG. 1: Possible structures for the magic number clusters $\mathrm{Sr}_{34}, \mathrm{Sr}_{61}$ and $\mathrm{Sr}_{82}$ along with their respective point groups.

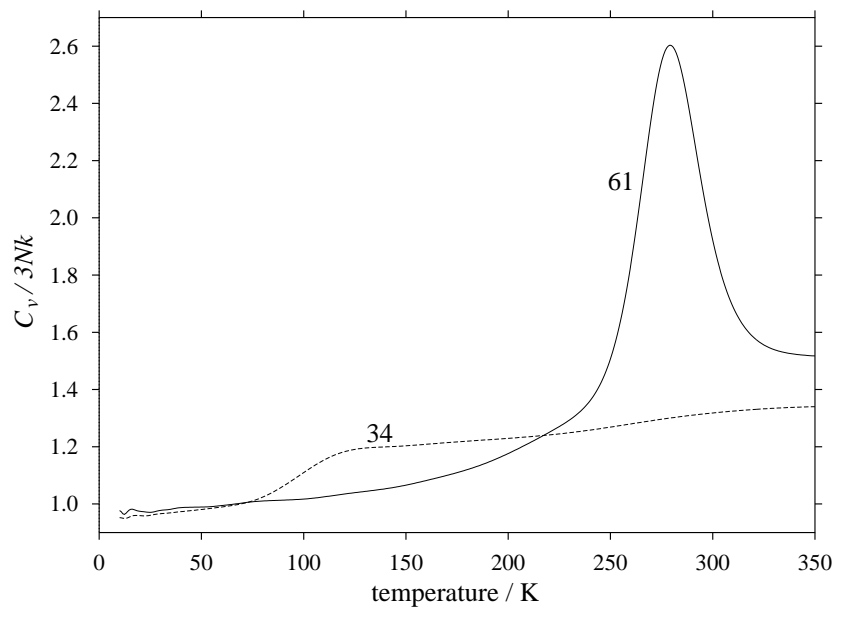

FIG. 2: Classical heat capacities for $\mathrm{Sr}_{34}$ and $\mathrm{Sr}_{61}$ calculated from parallel tempering simulations.

Furthermore, quenches from configurations generated by the simulations provided definitive evidence that there is no structural transition to the polytetrahedral structures of Fig. 1 (or to defective structures based upon them). For example, for $\mathrm{Sr}_{61}$ at $T=300 \mathrm{~K}$ the $T_{d}$ minimum was found only once in 4000 quenches, compared to 59 times for the global minimum; even above the melting temperature of this cluster, there is no sign of the relative stabilities of the two structures reversing. We confirmed that these results do not reflect ergodicity problems by running simulations starting from the $D_{5 h}$ and $T_{d}$ structures: the clusters quickly escaped from these structures, seldom to return.

To identify the cause of the differences between our results and those of Ref. 1, we repeated Wang et al.'s calculations of the partition functions of the global minima and the $D_{5 h}$ and $T_{d}$ structures using the harmonic approximation. However, we found the same scenario; i.e. structural transitions driven by the much greater vibrational entropy of the polytetrahedral structures. That the $D_{5 h}$ and $T_{d}$ structures are observed much less in the simulations than one would expect from these calculations, therefore points to a breakdown of the harmonic approximation as the cause of the huge overestimation of the partition functions of the $D_{5 h}$ and $T_{d}$ structures. This unusual behaviour - the harmonic approximation usually gives a correct qualitative picture of 
the thermodynamics - is worthy of further investigation. It may be that the small mean vibrational frequencies of the $D_{5 h}$ and $T_{d}$ structures are indicative of low barriers and basis of attractions that are small in extent.

Our results do not necessarily imply that the structures in Fig. 1 are incorrect assignments for the experimental magic numbers. Although our calculations with Wang et al.'s strontium potential are unable to provide any evidence in support of these assignments, this may instead reflect deficiencies in this potential and the difficulty of reliably modelling the structure of metal clusters.

1 G. M. Wang, E. Blaisten-Barojas, A. E. Roitberg, and T. P. Martin, J. Chem. Phys. 115, 3640 (2001).

2 J. P. K. Doye and D. J. Wales, Phys. Rev. Lett. 86, 5719 